\title{
Management of oncological patients in the digital era: anatomic pathology and nuclear medicine teamwork
}

\author{
Manuel Scimeca ${ }^{1,2,3}$, Nicoletta Urbano ${ }^{4}$, Rita Bonfiglio ${ }^{1}$, Orazio Schillaci ${ }^{1,5}$ \& Elena \\ Bonanno*,6,7 \\ 'Department of Biomedicine \& Prevention, University of Rome 'Tor Vergata', Via Montpellier 1, Rome 00133, Italy \\ ${ }^{2}$ IRCCS San Raffaele, Via di Val Cannuta 247, Rome 00166, Italy \\ ${ }^{3}$ OrchideaLab S.r.l., Via del Grecale 6, Morlupo, Rome (RM) 000674, Italy \\ ${ }^{4}$ Nuclear Medicine, Policlinico 'Tor Vergata', Rome 001335, Italy \\ ${ }^{5}$ IRCCS Neuromed, Pozzilli 860777, Italy \\ ${ }^{6}$ Department of Experimental Medicine \& Surgery, University 'Tor Vergata', Via Montpellier 1, Rome 00133, Italy \\ ${ }^{7}$ IRCSS Neuromed Lab. 'Diagnostica Medica' \& 'Villa dei Platani', Avellino 83100, Italy \\ *Author for correspondence: Tel.: +39062090 3913; elena.bonanno@uniroma2.it
}

"Specifically, the introduction of digital pathology in the clinical histological laboratories can lay the foundation of a successful creation of teamwork between anatomic pathology and nuclear medicine. In fact, image processing, analysis and interpretation represent essential steps in the activity of both these biomedical disciplines."

First draft submitted: 14 December 2017; Accepted for publication: 18 December 2017; Published online: 6 April 2018

Keywords: anatomic pathology • cancer • digital pathology • molecular imaging • nuclear medicine

Personalized medicine is one of the main objectives of both basic and translational research [1]. Nevertheless, it has become clear that the creation of personalized therapeutic protocols requires synergistic, transdisciplinary competencies. Indeed, new approved therapies rarely take into account both the interindividual variability and the aptitude of cancer cells to undergo those genetic and molecular adaptations involved in drug-resistance phenomenon. Thus, although promising biomedical discoveries have been made, the setup of 'patient-tailored' medical care is still far from becoming reality.

More often, researchers publish or patent molecules 'advertised' as powerful new 'weapons' for the fight against human cancers. Despite this, molecules that overcome preclinical trial steps are rarely truly translatable to the clinical side for diagnostic or therapeutical purposes. The discrepancy between experimental data and the possible use in both diagnosis and therapy of new anticancer molecules is due to several causes: biological differences between human diseases and animal models, inconsistence of experimental plans and/or wrong interpretation of the results. On note, in several studies no validation of data is performed by pathologists with long-term experience in cancer animal models.

However, new technologies applied on histological samples, as digital pathology, can provide the support to develop multidisciplinary studies based on digital imaging analysis. Specifically, the introduction of digital pathology in the clinical histological laboratories can lay the foundation of a successful creation of teamwork between anatomic pathology and nuclear medicine. In fact, image processing, analysis and interpretation represent essential steps in the activity of both these biomedical disciplines [2,3].

Already since the 1990s, imaging diagnostic departments, both radiology and nuclear medicine units, have been deeply transformed by the introduction of digital imaging techniques, with a resulting enhancement in quality and safety of reporting and innovation in the analysis and manipulation of diagnostic images [4,5]. In addition, imaging techniques are frequently used for translational research thanks to the development and dissemination of small-animal machines such as micro-PET/single photon emission computed tomography (SPECT). Conversely, digital pathology could offer similar benefits in the field of anatomic pathology.

Future Medicine 
Digital pathology and whole slide imaging have the potential to transform the histopathological data more and more 'real', quantifiable and comparable to that of other disciplines such as nuclear medicine. Despite the promise of digital pathology, its uptake in clinical and research practices has been slow.

Today, digital pathology is mainly used for the evaluation of prognostic and predictive immunohistochemical markers. Image analysis software is already widely available, and has the US FDA regulatory approval in the USA for the quantification of nuclear markers such as estrogen receptor (ER) or cell membrane markers such as HER2 [4,6].

This is important in the management of breast cancer patients since the treatment is actually based on immunohistochemical analysis of ER, progesterone receptor, Ki67 and HER2 [7]. Despite the improvement of the concordance between diagnosis and response to therapy, immunohistochemical analysis cannot capture the known spatial heterogeneity of breast cancer defined at the intratumoral, intrametastatic and intermetastatic level. Even though the temporal heterogeneity of cancer can be evaluated in sequential biopsies, this could imply the necessity of very invasive and repeated procedures. Thus, the quantification of immunohistochemical data can be used to design new PET-CT diagnostic or therapeutic protocols that improve the patients 'quality of life' by: reducing the number of biopsies, increasing the accuracy of the diagnosis and identifying in 'real time' the molecular transformation of cancerous cells. In particular, the use of radiolabeled molecules capable to quantify the expression of ER (i.e., 16 $\alpha$-18F-fluoro-17 $\beta$-estradiol) or cerb2 (i.e., radiolabeled anti-HER2 antibodies) by PET/CT could represent an alternative and reliable method for the management of breast cancer patients [8]. Even more important, molecular imaging analysis might improve the selection of patients for anti-HER2 therapy through systematic assessment and dynamic monitoring of HER2 expression in primary lesion, metastatic sites and circulating tumor cells.

Similarly, the research of new biomarkers of prostate cancer is a research field in constant development. Imaging of molecular prostate cancer has been recognized as a widely accepted clinical indication for prostate-specific membrane antigen (PSMA) ligand PET/CT in several parts of the world due to the results of numerous retrospective studies that indicate superior detection efficacy compared with standard of care imaging. The comparative expression of PSMA on prostate biopsies with digital pathology can further improve the accuracy of nuclear medicine analysis providing elements for both identification of new PSMA inhibitors and the comprehension of biological mechanisms related to PSMA expression in prostate lesions. In this context, Bernacki et al. demonstrated that antiPSMA immunohistochemical analysis is a promising marker for the identification of metastatic prostate carcinoma in surgical specimens [9].

The possibility to characterize histological tissues from ultrastructural and microanalytical point of view provides a further component for the development of a shared work platform between anatomic pathology and nuclear medicine. In particular, the use of transmission electron microscopy and scanning electron microscopy allows both proteins (immunogold labeling) [10] and chemical elements (microanalysis) to be identified at the subcellular level [11,12]. Taking advantage of this, the examination of bioptic samples of patients subjected to PET-CT investigation can provide information about quantification of PET-CT targets or even the exact localization of the radiolabeled molecules in the tissues (e.g., microanalysis can show the site of the link of radiolabeled molecules on tissues detecting the main elements of which they are composed such as gallium or technetium). By combining these data, it will be possible to increase the understanding of subcellular biodistribution of radiolabeled molecules and the biological mechanisms related to unspecific PET signals.

Another mutual contribution between these disciplines is the identification of a new cell type responsible for cancer occurrence and development. Recently, our research group described for the first time a new breast cancer cell type involved in microcalcification production, cancer growth and bone metastasis formation, the breast osteoblastlike cells (BOLCs) $[13,14]$. Detailed immunocharacterization of BOLCs both at the primary lesions and at the metastatic sites can provide the scientific rationale for the planning of preclinical experiments for the development and the synthesis of anti-BOLC-radiolabeled molecules able to identify/block this new cell type during bone metastasis formation. Also, molecular imaging analysis can guide immunotherapeutic strategies to enhance the efficacy of immune modulation therapies - for example, the anti-PD-L1 therapeutic protocols [15].

The dissertation here reported highlights the precious contribution offered by a close alliance between anatomic pathology and nuclear medicine to the scientific community. In particular, the construction of a structured collaboration model between these disciplines can speed up the achievement of a medicine that takes into account the uniqueness of the human being. 


\section{Financial \& competing interests disclosure}

The authors have no relevant affiliations or financial involvement with any organization or entity with a financial interest in or financial conflict with the subject matter or materials discussed in the manuscript. This includes employment, consultancies, honoraria, stock ownership or options, expert testimony, grants or patents received or pending, or royalties.

No writing assistance was utilized in the production of this manuscript.

\section{References}

1. Schillaci O, Urbano N. Personalized medicine: a new option for nuclear medicine and molecular imaging in the third millennium. Eur. J. Nucl. Med. Mol. Imaging 44(4), 563-566 (2017).

2. Van de Wiele C, Lahorte C, Oyen W et al. Nuclear medicine imaging to predict response to radiotherapy: a review. Int. J. Radiat. Oncol. Biol. Phys. 55(1), 5-15 (2003).

3. Saco A, Ramírez J, Rakislova N, Mira A, Ordi J. Validation of whole-slide imaging for histolopathogical diagnosis: current state. Pathobiology 83(2-3), 89-98 (2017).

4. Griffin J, Treanor D. Digital pathology in clinical use: where are we now and what is holding us back? Histopathology 70(1), 134-145 (2017).

5. Schillaci O. Use of dual-point fluorodeoxyglucose imaging to enhance sensitivity and specificity. Semin. Nucl. Med. 42(4), 267-280 (2012).

6. US FDA. FDA 510(k) clearances. https://digitalpathologyassociation.org/_-data/files/DPA_Regulatory-FDA-510k_list.pdf

7. Senkus E, Kyriakides S, Ohno S et al. Primary breast cancer: ESMO Clinical Practice Guidelines for diagnosis, treatment and follow-up. Ann. Oncol. 26 (Suppl. 5), v8-v30 (2015).

8. Capala J, Bouchelouche K. Molecular imaging of HER2-positive breast cancer: a step toward an individualized 'image and treat' strategy. Curr. Opin. Oncol. 22(6), 559-566 (2015).

9. Bernacki KD, Fields KL, Roh MH. The utility of PSMA and PSA immunohistochemistry in the cytologic diagnosis of metastatic prostate carcinoma. Diagn. Cytopathol. 42(7), 570-575 (2014).

10. Scimeca M, Antonacci C, Colombo D, Bonfiglio R, Buonomo OC, Bonanno E. Emerging prognostic markers related to mesenchymal characteristics of poorly differentiated breast cancers. Tumour Biol. 37(4), 5427-5435 (2016).

11. Scimeca M, Pietroiusti A, Milano F et al. Elemental analysis of histological specimens: a method to unmask nano asbestos fibers. Eur. J. Histochem. 60(1), 2573 (2016).

12. Scimeca M, Orlandi A, Terrenato I, Bischetti S, Bonanno E. Assessment of metal contaminants in non-small cell lung cancer by EDX microanalysis. Eur. J. Histochem. 58(3), 2403 (2014).

13. Scimeca M, Antonacci C, Toschi N et al. Breast osteoblast-like cells a reliable early marker for bone metastases from breast cancer. Clin. Breast Cancer doi: 10.1016/j.clbc.2017.11.020 (2017) (Epub ahead of print).

14. Scimeca M, Bonfiglio R, Montanaro M, Bonanno E. Osteoblast-like cells in human cancers: new cell type and reliable markers for bone metastasis. Future Oncol. 14(1), 9-11 (2018)

15. Bonfiglio R, Nardozi D, Scimeca M, Cerroni C, Mauriello A, Bonanno E. PD-L1 in immune-escape of breast and prostate cancers: from biology to therapy. Future Oncol. 13(24), 2129-2131 (2017). 
